\title{
Search for CPT and Lorentz Symmetry Violation in Neutral Kaons at KLOE/KLOE-2
}

\section{Antonio De Santis* on behalf of KLOE-2 collaboration}

E-mail: antonio.desantis@romal.infn.it

The KLOE experiment at the DA $\Phi N E \phi$-factory of the INFN Frascati Laboratory collected data corresponding to $2.5 \mathrm{fb}^{-1}$ of integrated luminosity. Neutral kaon pairs produced in $\phi$ decays offers a unique possibility to perform tests of fundamental discrete symmetries. In this contribution the entanglement of the two kaons is exploited to search for possible violation of CPT symmetry and Lorentz invariance in the context of the Standard-Model Extension (SME) framework. A new approach to the analysis of $\phi \rightarrow K_{S} K_{L} \rightarrow \pi^{+} \pi^{-}, \pi^{+} \pi^{-}$events has been adopted allowing us to independently measure all four CPT violating parameters $\Delta a_{\mu}$ appearing for neutral kaons in the SME. The final KLOE results on $\Delta a_{\mu}$ are presented:

$$
\begin{aligned}
& \Delta a_{0}=\left(-6.0 \pm 7.7_{\text {stat }} \pm 3.1_{\text {syst }}\right) \times 10^{-18} \mathrm{GeV} \\
& \Delta a_{X}=\left(0.9 \pm 1.5_{\text {stat }} \pm 0.6_{\text {syst }}\right) \times 10^{-18} \mathrm{GeV} \\
& \Delta a_{Y}=\left(-2.0 \pm 1.5_{\text {stat }} \pm 0.5_{\text {syst }}\right) \times 10^{-18} \mathrm{GeV} \\
& \Delta a_{Z}=\left(3.1 \pm 1.7_{\text {stat }} \pm 0.5_{\text {syst }}\right) \times 10^{-18} \mathrm{GeV}
\end{aligned}
$$

We also shortly discuss the perspectives for a new measurement using the KLOE-2 apparatus equipped with a new inner tracker.

2013 Kaon Physics International Conference, 29 April-1 May 2013

University of Michigan, Ann Arbor, Michigan - USA

\section{*Speaker.}

${ }^{\dagger}$ D. Babusci, D. Badoni, I. Balwierz-Pytko, G. Bencivenni, C. Bini, C. Bloise, F. Bossi, P. Branchini, A. Budano, L. Caldeira Balkestå, G. Capon, F. Ceradini, P. Ciambrone, F. Curciarello, E. Czerwiński, E. Danè, V. De Leo, E. De Lucia,G. De Robertis, A. De Santis, A. Di Domenico, C. Di Donato, R. Di Salvo, D. Domenici, O. Erriquez, G. Fanizzi, A. Fantini, G. Felici, S. Fiore, P. Franzini, A. Gajos, P. Gauzzi, G. Giardina, S. Giovannella, F. Gonnella, E. Graziani, F. Happacher, L. Heijkenskjöld, B. Höistad, L. Iafolla, M. Jacewicz, T. Johansson, K. Kacprzak, A. Kupsc, J. LeeFranzini, B. Leverington, F. Loddo, S. Loffredo, G. Mandaglio, M. Martemianov, M. Martini, M. Mascolo, R. Messi, S. Miscetti, G. Morello, D. Moricciani, P. Moskal, F. Nguyen, A. Palladino, A. Passeri, V. Patera, I. Prado Longhi, A. Ranieri, C. F. Redmer, P. Santangelo, I. Sarra, M. Schioppa, B. Sciascia, M. Silarski, C. Taccini, L. Tortora, G. Venanzoni, W. Wiślicki, M. Wolke, J. Zdebik 


\section{The KLOE experiment}

The KLOE experiment operates at DAФNE, the Frascati $\phi$-factory. DA $\Phi$ NE is an $e^{+} e^{-}$ collider running at a center of mass energy of $\sim 1020 \mathrm{MeV}$, the mass of the $\phi$ meson. Positron and electron beams collide at an angle of $\pi-25 \mathrm{mrad}$, producing $\phi$ mesons with small momentum in the orbit plane $\left(p_{x}(\phi) \sim-15 \mathrm{MeV}\right)$.

The KLOE detector consists of a large cylindrical drift chamber (DC) surrounded by a leadscintillating fiber electromagnetic calorimeter (EMC). A super-conducting coil around the EMC provides a $0.52 \mathrm{~T}$ axial field.

The DC[1] is $4 \mathrm{~m}$ in diameter and $3.3 \mathrm{~m}$ long and has 12,582 all-stereo tungsten sense wires. The chamber shell is made of carbon fiber composite and the gas used is a $90 \%$ helium, $10 \%$ isobutane mixture. These features maximize transparency to photons and reduce $K_{L} \rightarrow K_{S}$ regeneration and multiple scattering. The position resolutions for single hits are $\sigma_{r, \phi} \sim 150 \mu \mathrm{m}$ and $\sigma_{z} \sim 2 \mathrm{~mm}$ almost omogeneus in the active area. The momentum resolution is $\sigma\left(p_{\perp}\right) / p_{\perp} \sim 0.4 \%$.

The calorimeter[2] is divided into a barrel and two end-caps, and covers $98 \%$ of the solid angle. The modules are read-out at both ends by photo-multipliers, both in amplitude and time for a total of 2440 cells per side arranged in five layers. Cells close in time and space are grouped into calorimeter clusters. The cluster energy $E$ is the sum of the cell energies. The cluster time $T$ and position $\vec{R}$ are energy-weighed averages. Energy and time resolutions are $\sigma_{E} / E=5.7 \% / \sqrt{E(\mathrm{GeV})}$ and $\sigma_{t}=57 \mathrm{ps} / \sqrt{E(\mathrm{GeV})} \oplus 100 \mathrm{ps}$, respectively.

During KLOE data taking DA $\Phi$ NE beam conditions and detector calibrations are constantly monitored in order to guarantee the highest quality of the collected data. Presently KLOE has acquired $2.5 \mathrm{fb}^{-1}$ of data and a new extensive campaign of data taking is starting under the project KLOE-2 [3].

\section{Neutral kaons at KLOE}

At DAФNE the $\phi$ is produced almost at rest in the center of the KLOE detector and decay mostly in kaon pairs: $34 \%$ of decays are neutral kaons. The initial state of the kaon pair is produced with quantum numbers $J^{P C}=1^{--}$:

$$
|i\rangle=\frac{\left|K_{0}\right\rangle\left|\bar{K}_{0}\right\rangle-\left|\bar{K}_{0}\right\rangle\left|K_{0}\right\rangle}{\sqrt{2}}=\mathscr{N}\left(\left|K_{S}(\vec{p})\right\rangle\left|K_{L}(-\vec{p})\right\rangle-\left|K_{S}(-\vec{p})\right\rangle\left|K_{L}(\vec{p})\right\rangle\right),
$$

where $\left|K_{S} / K_{L}\right\rangle$ are the kaon mass eigenstates and $\mathscr{N}$ is a normalization factor.

As usual the neutral kaon mass eigenstates could be expressed as:

$$
\left\{\begin{array}{l}
\left|K_{S}\right\rangle=\left[\left(1+\varepsilon_{S}\right)\left|K_{0}\right\rangle+\left(1-\varepsilon_{S}\right)\left|\bar{K}_{0}\right\rangle\right] / N_{S} \\
\left|K_{L}\right\rangle=\left[\left(1+\varepsilon_{L}\right)\left|K_{0}\right\rangle-\left(1-\varepsilon_{L}\right)\left|\bar{K}_{0}\right\rangle\right] / N_{L}
\end{array}\right.
$$

where $\varepsilon_{S / L}=\varepsilon_{K} \pm \delta_{K}$ and $N_{S / L}$ are normalization factors. The parameter $\varepsilon_{K}$ represents $C P$ violation in the neutral kaon mixing while $\delta_{K}$ stands for possible $C P T$ violation. Assuming no $C P T$ violation $\varepsilon_{S}=\varepsilon_{L}$.

Equation 2.1 implies that the detection of one of the two kaons on one side reveals the presence of the other kaon on the opposite side. In the KLOE data reconstruction this feature is used to select pure $K_{S}$ or $K_{L}$ beams identifying $K_{L}$ interactions in the EMC or $K_{S} \rightarrow \pi^{+} \pi^{-}$decays respectively. 
A different approach can be exploited at KLOE since the two kaons are produced in an antisymmetric correlated state. Labeling $f_{1}$ and $f_{2}$ the decay channels for the two kaons, the time evolution of the initial state decaying into $\left|f_{1}, f_{2}\right\rangle$ final state is expressed as a function of the difference of proper decay times $\left(\Delta \tau=\tau_{2}-\tau_{1}\right)$ as:

$$
I_{f_{1} f_{2}}(\Delta \tau) \propto e^{-\Gamma|\Delta \tau|}\left[\left|\eta_{f_{1}}\right|^{2} e^{\frac{\Delta \Gamma}{2} \Delta \tau}+\left|\eta_{f_{2}}\right|^{2} e^{-\frac{\Delta \Gamma}{2} \Delta \tau}-2 \Re e\left(\eta_{f_{1}} \eta_{f_{2}}^{*} e^{-i \Delta m \Delta \tau}\right)\right]
$$

where $\eta_{f_{j}}=\left\langle f_{j} \mid K_{L}\right\rangle /\left\langle f_{j} \mid K_{S}\right\rangle, \Gamma=\Gamma_{S}+\Gamma_{L}$ and $\Delta \Gamma=\Gamma_{S}-\Gamma_{L}$.

Equation 2.3 shows a time interference term characteristic of the type of correlation firstly pointed out by Einstein, Podolsky and Rosen [4].

If the considered final states are the same for the two kaons $\left(f_{1}=f_{2}=f\right)$ it becomes impossible to distinguish between them (e.g. decay times are not ordered). In the standard Quantum Mechanical description of the time evolution of the system, a fully destructive interference is expected for equal decay times $(|\Delta \tau|=0)$. Assuming $f_{j}=\pi^{+} \pi^{-}$the resulting ratio of amplitudes $\left(\eta_{j}\right)$ becomes:

$$
\eta_{j}=\eta_{\pi^{+} \pi^{-}}=\frac{\left\langle K_{L} \mid \pi^{+} \pi^{-}\right\rangle}{\left\langle K_{S} \mid \pi^{+} \pi^{-}\right\rangle} \simeq \varepsilon_{K}+\varepsilon^{\prime}-\delta_{K}
$$

In the Standard Model Extension (SME) framework developed by A. Kostelecky [5] the $\delta_{K}$ parameter is not constant. The general building principle of such a model is to construct the most general Lagrangian for SM particles and their interactions explicitly including all the possible terms violating CPT and Lorentz Invariance. The constraint applied to build the Lagrangian is to have a renormalizable theory fulfilling the unitarity and causality. This general assumptions follows the so-called anti-CPT or Greenberg theorem [6] for which is possible to have quantum field theory not fulfilling the CPT theorem only releasing the Lorentz Invariance hypothesis while keeping the unitarity and causality.

In SME framework the $\delta_{K}$ is expected to have the following structure:

$$
\delta_{K} \approx i \sin \phi_{S W} e^{i \phi_{S W}} \gamma_{K}\left(\Delta a_{0}-\vec{\beta}_{K} \cdot \Delta \vec{a}\right) / \Delta m,
$$

where $\gamma_{K}$ and $\beta_{K}$ are the usual Lorentz factors for the kaons , $\phi_{S W}$ is the super-weak phase and $\Delta a_{\mu}$ are the SME parameters for the kaon system.

Equation 2.5 shows that $\delta_{K}$ is modulated by the kaon momentum modulus $\left(\gamma_{K}\right.$ and $\left|\vec{\beta}_{K}\right|$ ) and by its spatial direction $\left(\vec{\beta}_{K}\right)$. In the KLOE case the two kaons are produced almost back-to-back in the $\phi$ decay and therefore evolve with two different $\delta_{K}\left(\delta_{K}\left(\vec{P}_{1}\right) \neq \delta_{K}\left(\vec{P}_{2}\right)\right)$. The effect produced by CPT violation can be observed in the distribution of equation 2.3 provided that the two kaon final states are tagged with respect privileged reference frame.

Taking into account the Earth motion with respect to fixed stars, and local coordinate of the KLOE detector the $C P T$ violation parameter becomes:

$$
\begin{aligned}
\delta_{K}\left(\vec{P}_{K}, T_{\text {sid }}\right)= & \frac{i \sin \phi_{S W} e^{i \phi_{S W}}}{\Delta m} \gamma_{K}\left[\Delta a_{0}+\beta_{K} \Delta a_{Z}(\cos \vartheta \cos \chi-\sin \vartheta \cos \varphi \sin \chi)\right. \\
& -\beta_{K} \Delta a_{X} \sin \vartheta \sin \varphi \sin \omega_{E} T_{\text {sid }} \\
& +\beta_{K} \Delta a_{X}(\cos \vartheta \sin \chi+\sin \vartheta \cos \varphi \cos \chi) \cos \omega_{E} T_{\text {sid }} \\
& +\beta_{K} \Delta a_{Y}(\cos \vartheta \sin \chi+\sin \vartheta \cos \varphi \cos \chi) \sin \omega_{E} T_{\text {sid }} \\
& \left.+\beta_{K} \Delta a_{Y} \sin \vartheta \sin \varphi \cos \omega_{E} T_{\text {sid }}\right]
\end{aligned}
$$


where $\omega_{E}$ is the angular velocity of the Earth, $T_{\text {sid }}$ is the sidereal time, $\chi$ is the $3 \mathrm{D}$ angle between the $\hat{z}_{L A B}$ axis of the laboratory frame and the Earth rotation axis and $\vartheta$ and $\varphi$ are the polar and azimuthal angle in the laboratory, respectively. The different reference frame are shown in figure 1.
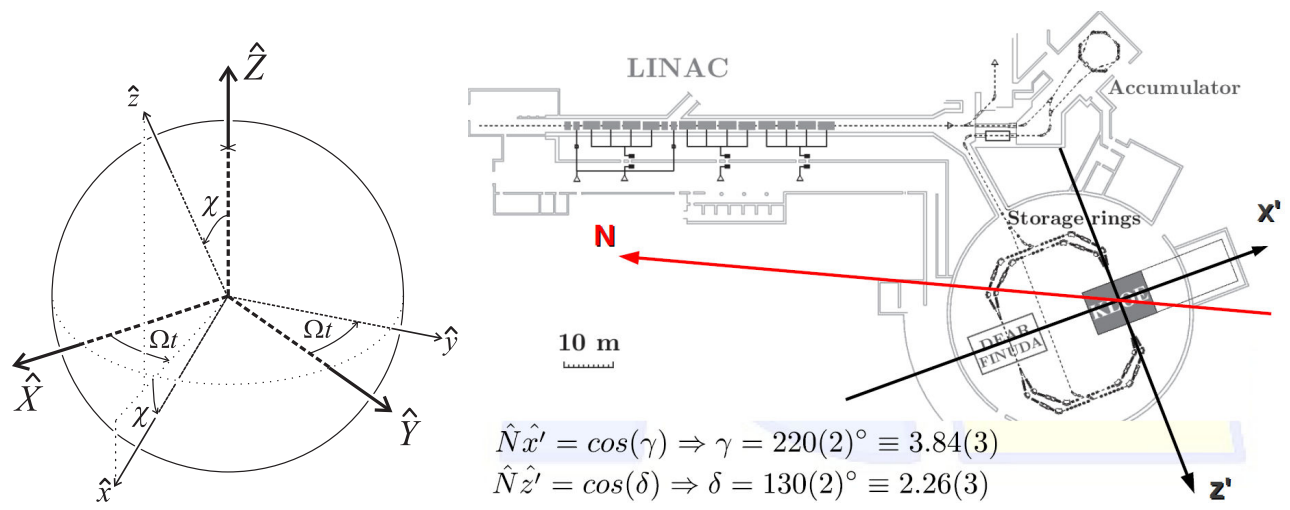

Figure 1: Fixed and rotating reference frame (left). Notice that is required that the $\hat{x}$ axis lie in the plane generated from $\hat{Z}$ and $\hat{z}$. Kloe reference frame with respect to Earth coordinates. DAФNE complex and KLOE site description (right). The KLOE reference frame axes are shown. The north direction (in red) has been derived using a compass inside the DAФNE hall. Accuracy of this method has been estimated to be 1-2 degree. The KLOE reference frame has to be rotated by an angle $\phi_{R}=130^{\circ}$ to be the one of equation 2.6

The introduction of equation 2.6 in the time evolution of the kaon system implies the angular dependence of equation 2.3:

$$
\begin{aligned}
I\left(\Delta t, T_{\text {sid }}, \vartheta_{K_{1}}, \varphi_{K_{1}}\right) \propto & e^{-\Gamma|\Delta \tau|}\left[\left|\varepsilon_{K}-\delta_{K}\left(\vec{P}_{1}\right)\right|^{2} e^{\frac{\Delta \Gamma}{2} \Delta \tau}+\right. \\
& \left|\varepsilon_{K}-\delta_{K}\left(\vec{P}_{\phi}-\vec{P}_{1}\right)\right|^{2} e^{-\frac{\Delta \Gamma}{2} \Delta \tau}- \\
& 2 \Re e\left(\left(\varepsilon_{K}-\delta_{K}\left(\vec{P}_{1}\right)\left(\varepsilon_{K}-\delta_{K}\left(\vec{P}_{\phi}-\vec{P}_{1}\right)\right)^{*} e^{-i \Delta m \Delta \tau}\right)\right]
\end{aligned}
$$

where $\vartheta_{K_{1}}, \varphi_{K_{1}}$ are the polar and azimuthal angle for one of the two kaons ordered according to the their momentum direction, respectively.

Since at KLOE the $\phi$ is not produced exactly at rest the kaons are not perfectly opposite in the lab frame. This has two implications: the Lorentz factors in equation $2.6\left(\gamma_{K}\right.$ and $\left.\beta_{K}\right)$ have their own angular dependence and the two kaons momenta are not equal in modulus.

The equation 2.6 shows that the sidereal time dependence of $\delta_{K}$ could be observed only associated with $\Delta a_{X}$ and $\Delta a_{Y}$ parameters different from zero. $\Delta a_{0}$ parameter, being coupled only with the $\gamma_{K}$, will be the most difficult to observe ${ }^{1}$.

In order to measures $\Delta a_{\mu}$ one needs to analyze the KLOE data-set as a function of sidereal time and kaon angle. The kaon ordering according to the sign of momentum component along $\mathrm{z}$ reflects in the $\Delta \tau$ sign. To be sensitive to the $\Delta a_{0}$ parameter effects, the azimuthal angle dependence is considered by splitting the sample according to kaon direction with respect to $\phi$ momentum (e.g.

\footnotetext{
${ }^{1}$ The variation of $\gamma_{K}$ at KLOE is of the order of $2-3 \%$.
} 
$P_{x}>0$ or $\left.P_{x}<0\right)$. The observable will be defined as:

$$
S\left(\Delta \tau_{i}, \Delta T_{\text {sid } j}, \Delta \Omega_{h}\right)=S_{i j h}=\int_{\Delta \tau_{i}} d \Delta \tau \int_{\Delta T_{\text {sid } j}} d T_{\text {sid }} \int_{\Delta \Omega_{h}} d \Omega_{K_{1}} \rho\left(\Omega_{K_{1}}, T_{\text {sid }}\right) I\left(\Delta \tau, T_{\text {sid }}, \Omega_{K_{1}}\right)
$$

where $\rho\left(\Omega_{K_{1}}, T_{\text {sid }}\right)$ is the angular and sidereal density distribution of the produced kaon in the positive direction of the $\mathrm{z}$ axis. This density is expected to be flat in $T_{\text {sid }}$ and azimuthal angle while should have $\sin ^{3} \vartheta$ dependence in the polar angle. The integration ranges are defined as:

$\Delta \tau_{i}$ Proper decay time difference. Bin width $1 \tau_{S}$

$\Delta T_{\text {sid } j}$ Sidereal time interval. The default number of divisions of a sidereal day will be four, 6 sidereal hour each;

$\Delta \Omega_{h}$ Angular interval. The polar angle range will be $\vartheta \in[0, \pi / 2[$ and the azimuthal range will be $\varphi \in[-\pi / 2 ; \pi / 2[$ or $\varphi \in[\pi / 2 ; 3 \pi / 2[$, sel. "A" and sel. "B", respectively.

The number of different $\Delta \tau$ distribution considered in the present analysis will be $8: 4$ sidereal time bins $\times 2$ azimuthal angular bins.

Starting from the theoretical expression of equation 2.8 the observed distribution $\left(\tilde{S}\left(\Delta \tau_{i}, \Delta T_{s i d j}, \Delta \Omega_{h}\right)\right)$ will be comprehensive of efficiency and resolution effects:

$$
\tilde{S}\left(\Delta \tau_{i}, \Delta T_{\text {sid } j}, \Delta \Omega_{h}\right)=\tilde{S}_{i, j, h}=\rho_{\mathrm{K}_{\mu} 3}\left(\Delta \tau_{i}\right)\left(1+f_{\text {regen }}\left(\Delta \tau_{i}\right)\right) \sum_{k} \varepsilon_{k}^{M C} P_{k i}^{M C} S_{k, j, h}
$$

where $\rho_{\mathrm{K}_{\mu} 3}\left(\Delta \tau_{i}\right)$ is the efficiency correction from a semileptonic control sample that take into account difference between data and $\mathrm{MC}$ in the efficiency for triggering and reconstruction, $f_{\text {regen }}\left(\Delta \tau_{i}\right)$ is a correction factor that takes into account the kaon regeneration on the beam pipe structures, $\varepsilon_{k}^{M C}$ is the total efficiency corresponding to the $\mathrm{MC}$ bin $\left(\Delta \tau_{k}^{M C}\right)^{2}$ and $P_{i k}^{M C}$ is the smearing matrix. The kaon regeneration at the beam pipe is taken into account in $\tilde{S}\left(\Delta \tau_{i}, \Delta T_{s i d j}, \Delta \Omega_{h}\right)$ according to [7].

\section{Data analysis}

In this analysis the entire KLOE data-set acquired during 2004-05 has been used. The total integrated luminosity is about $1.7 \mathrm{fb}^{-1}$. The Monte-Carlo (MC) samples used are two, with an equivalent integrated luminosity of $3.4 \mathrm{fb}^{-1}$ and $17 \mathrm{fb}^{-1}$, respectively. The former, containing all the $\phi$-meson decay channels, has been used for analysis optimization, while the latter, containing only signal events has been used for efficiency and resolution determination.

The data reduction starts with the topological identification of the candidate signal events: two vertices with two tracks each. For each vertex the same list of selection criteria is applied:

- $\left|M_{t r k}-M_{K}\right|<5 \mathrm{MeV}$

where $M_{t r k}$ is the invariant mass of the kaon reconstructed from the tracks assuming charged pion mass hypothesis;

\footnotetext{
${ }^{2}$ The difference between data and MC $\Delta \tau$ bins is simply related to the width. To have a nice smoothing in the smearing the data bin width is a multiple of the one used for MC.
} 
- $\sqrt{E_{\text {miss }}^{2}+\left|\vec{P}_{\text {miss }}\right|^{2}}<10 \mathrm{MeV}$

where $\vec{P}_{\text {miss }}=\vec{P}_{K}($ tag $)-\vec{P}_{K}($ trk $)$ with $\vec{P}_{K}($ tag $)=\vec{P}_{\phi}-\vec{P}_{K}$ (opposite) the kaon tagged momentum from the opposite decay vertex information and $\vec{P}_{K}(t r k)=\vec{p}_{\pi^{+}}+\vec{p}_{\pi^{-}}$the reconstructed kaon momentum from the tracks, $E_{\text {miss }}=E_{K}($ tag $)-E_{K}($ trk $)$ with $E_{K}($ tag $)=E_{\phi}-$ $E_{K}$ (opposite) the kaon tagged energy from the opposite decay vertex informations and $E_{K}(t r k)=\sqrt{\left|\vec{p}_{\pi^{+}}\right|^{2}+m_{\pi}^{2}}+\sqrt{\left|\vec{p}_{\pi^{-}}\right|^{2}+m_{\pi}^{2}}$ is the energy of the kaon reconstructed from tracks;

- $-50 \mathrm{MeV}^{2}<M_{\text {miss }}^{2}<10 \mathrm{MeV}^{2}$

where $M_{\text {miss }}^{2}=E_{\text {miss }}^{2}-\left|\vec{P}_{\text {miss }}\right|^{2}$

- $\mid P_{K}^{*}($ trk $)-P_{0}^{*}($ kinematics $) \mid<10 \mathrm{MeV}$

where $P_{K}^{*}(t r k)$ is the momentum of the kaon, as derived from tracks, in the $\phi$-meson reference frame, while $P_{0}^{*}($ kinematics $)=\sqrt{\frac{s^{2}}{4}-m_{K_{0}}^{2}}$ represents the expected value from two body kinematics in the $\phi$-meson decay.

The background contamination after those selections is $1.5 \%$, as estimated from MC. The background events are mainly due to kaon regeneration. This background is irreducible because is made of regenerated kaons moving in the forward direction (in the limit of the angular resolution).

A global fit is performed in order to improve the resolution on the decay time difference. In this global fit the free parameters are the $\phi$-meson decay point coordinates $\left(\vec{V}_{\phi}\right)$ and the decay length for the two kaons $\left(\lambda_{1,2}\right)$. A global Likelyhood function is built in order to take into account all the information for each event. The outputs of the fit are then used to compute the proper decay times and $\chi^{2}$ and pulls of the fit are used to reject badly reconstructed events.

The $\Delta \tau$ resolution is strongly correlated, as expected, with the opening angle of the pion tracks $\left(\vartheta_{ \pm}\right)$, and deteriorates at large values of $\vartheta_{ \pm}$. A cut to eliminate large opening angles values has been applied and events with: $\cos \left(\vartheta_{ \pm}\right)<-0.975$ have been rejected. The result is shown in figure 2. Since the two kaons are ordered according to the longitudinal momentum (z component) it is possible to mis-order them with respect to the true ordering. This effect has been suppressed by rejecting events with the "first" kaon having: $P_{z}\left(K_{1}\right)<2 \mathrm{MeV}$.

The shape of the efficiency as a function of true value of $\Delta \tau$ is shown in figure 2 . The mean value is $25 \%$ for $\Delta \tau$ ranging in $[-12 ; 12] \tau_{S}$ with a consistent drop for $\Delta \tau \sim 0$. This is due to two concurrent effects: increasing extrapolation length for tracks coming from the interaction point and the wrong association of tracks with the kaon decay vertex ${ }^{3}$ ("vertex interference").

The total selection efficiency can be parametrized as follow:

$$
\varepsilon_{t o t}=\varepsilon_{t r i g} \varepsilon_{\text {reco }} \varepsilon_{\text {cuts }}
$$

where the first two terms take into account trigger and standard reconstruction procedure effects while the last is the effect of the selection criteria. In this analysis the efficiencies are derived from MC. For trigger and reconstruction efficiencies corrections obtained from data, using an independent control sample of $K_{L} \rightarrow \pi \mu \nu$, are applied. The control sample is selected so to have the best purity and to cover the track momentum distribution of the signal.

\footnotetext{
${ }^{3}$ When the two vertex are so close in space $\left(\Delta \tau=1 \tau_{S} \Rightarrow \Delta x_{V} \sim 6 \mathrm{~mm}\right)$ it is possible to wrongly associate tracks to vertex by exchanging two of them.
} 

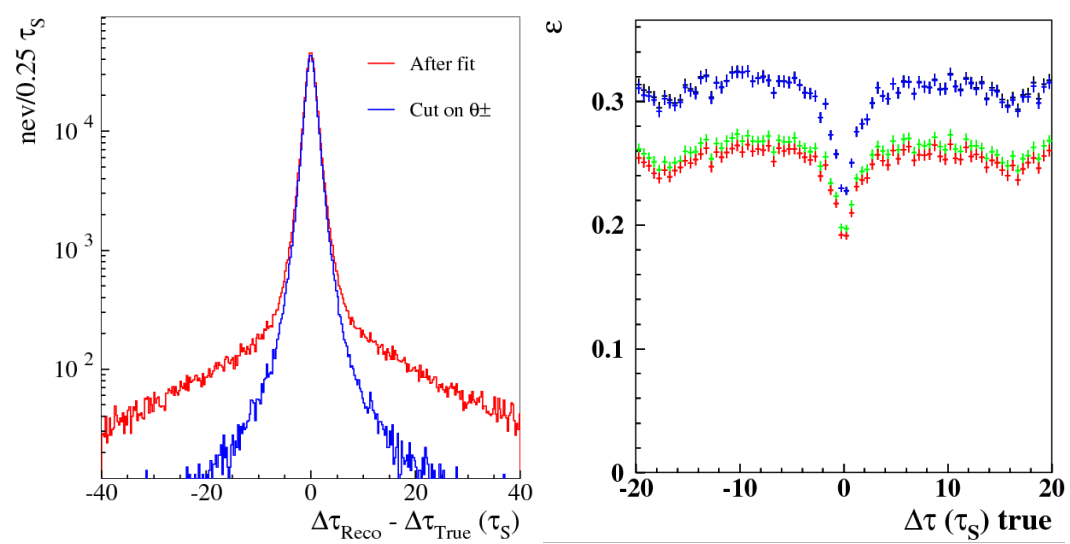

Figure 2: Left: resolution on $\Delta \tau$. The effect of the cut on opening angle between tracks is shown. Right: $\mathrm{MC}$ efficiency as a function of true $\Delta \tau$. Colors represents the different analysis stage: blue for global fit, green for opening angle cuts and red for the final efficiency.

The selection of the control sample requires the presence of a $K_{S} \rightarrow \pi^{+} \pi^{-}$tagging decay and a semileptonic decay on the opposite side. After the topological request (two tracks connected to a vertex), the selection of the semileptonic decay is mainly based on the following variable:

$$
m_{l}^{2}( \pm)=E_{l}^{2}-p_{ \pm}^{2}=\left(E_{K}^{t a g}-E_{\pi}(\mp)-\left|\vec{p}_{m i s s}\right|\right)^{2}-p_{ \pm}^{2}
$$

representing the reconstructed mass of the lepton of a given charge in the hypothesis that the other charged track is a pion. In figure 3 the distribution of the squared charged lepton mass is shown. The structures corresponding to $\mathrm{K}_{e} 3, \mathrm{~K}_{\mu} 3$ and $\pi^{+} \pi^{-}$decay are clearly visible. The control sample is required to fulfill:

$$
m_{l}^{2}(+)-m_{l}^{2}(-) \geq 15000 \mathrm{MeV}^{2}, \quad m_{l}^{2}(+)-m_{l}^{2}(-) \leq 30000 \mathrm{MeV}^{2}
$$

After this simple selection the purity of $\mathrm{K}_{\mu} 3$ sample is at the level of $93 \%$. To further improve the purity the following cuts are applied:

- $\sqrt{E_{\text {miss }}^{2}+P_{\text {miss }}^{2}}>10 \mathrm{MeV}$ to remove the residual $K_{L} \rightarrow \pi^{+} \pi^{-}$events ensuring the statistical independence between signal and control sample;

- $\left|p_{+}^{+}\right|+\left|p_{-}^{*}\right|<400 \mathrm{MeV}$

- $\cos \left(\vartheta_{ \pm}\right)<-0.6$ to ensure the same angular range for the opening angle between tracks. This last cut is applied also on the tagging side.

The final purity of the control sample is $95 \%$ and the remaining background is fully dominated by $\mathrm{K}_{e} 3$ decay. The efficiency correction has been evaluated as the ratio between data and $\mathrm{MC}$ distribution of $\Delta \tau$, as shown in figure 3 . 

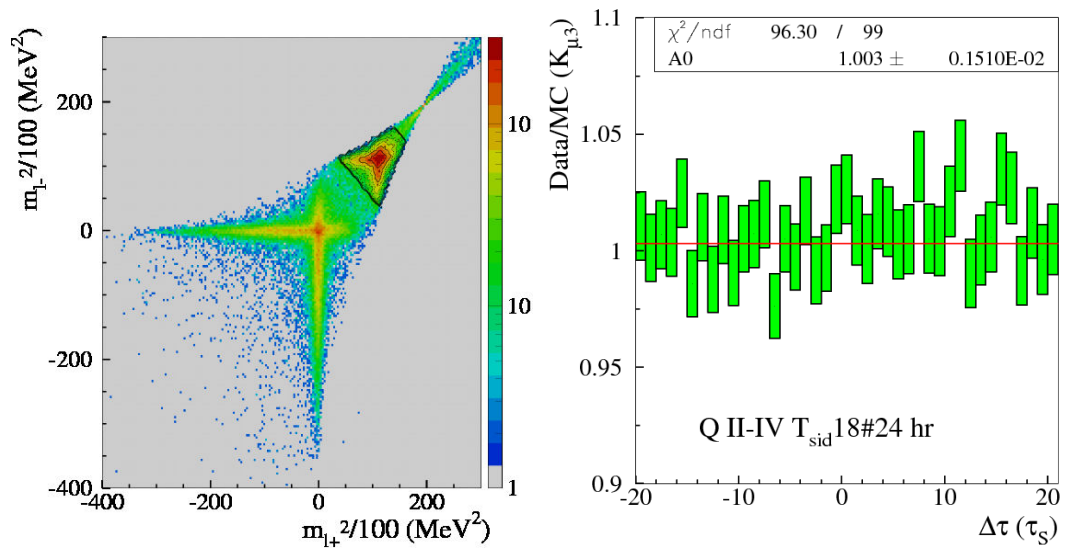

Figure 3: Left: squared charged lepton mass hypotheses correlation plot. The highlighted region represent the cuts applied. Right: Efficiency correction as a function of $\Delta \tau$ in the sidereal time interval 18-24 hours. Plot refers to the angular selection: sel. "B". Boxes represent statistical errors. The fit with a constant function shows that on average the global correction is very small $(0.2-0.5 \%)$.

\section{Fit results}

The data distributions selected as described in section 3 have been fitted with the SME theoretical function with the procedure described in section 2. The results obtained from the fit are listed in table 1 . The $\Delta \tau$ range is: $\Delta \tau \in[-12: 12] \tau_{S}$. This choice is to limit the perturbation on the result by the regeneration on the spherical beam pipe. The number of sidereal time bins used is four and the angular selection are two: $\cos \vartheta_{K_{1}}>0 \cos \varphi_{K_{1}}>0$ (sel. "A") and $\cos \vartheta_{K_{1}}>0 \cos \varphi_{K_{1}}<0$ (sel. "B"). All the distributions are fitted at the same time (184 bins).
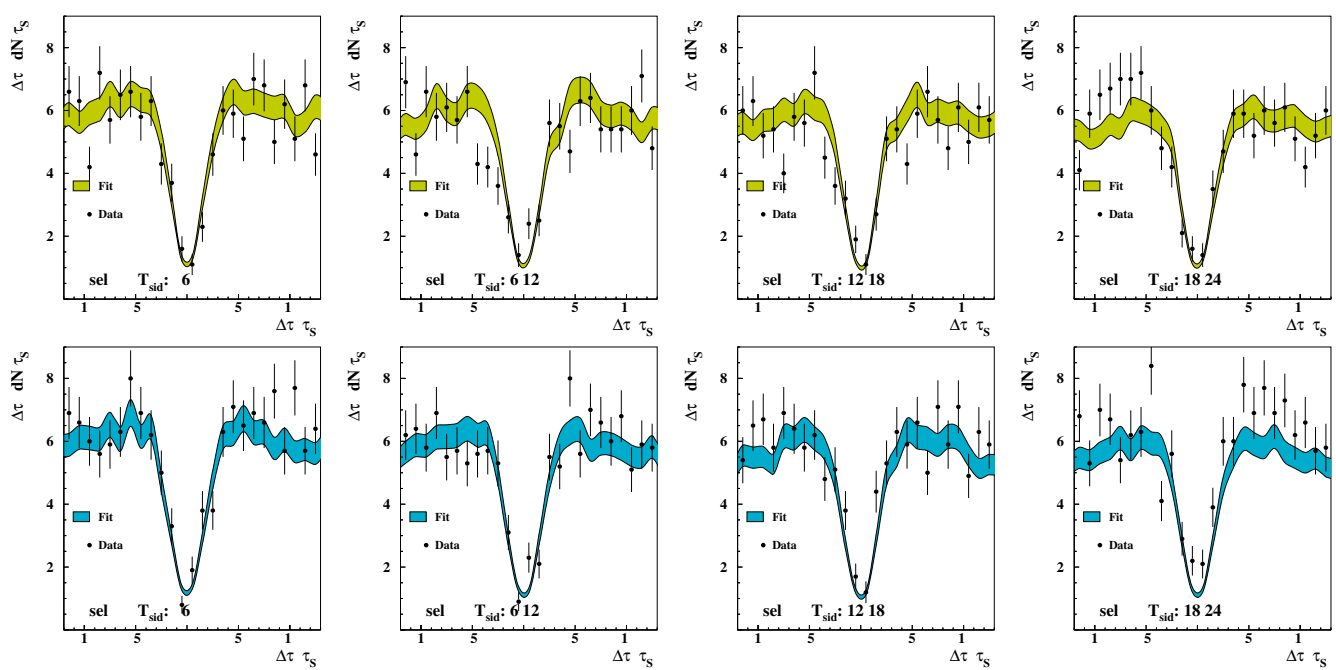

Figure 4: Fit results: upper(lower) plots are for the angular selection "A"("B") . Black points are for data while colored bands are the fit output. The errors on date are purely statistical, while the width of the fit result band represent the contribution to the uncertainty coming from MC statistics and efficiency correction. 
The systematic effects considered in the present analysis are:

Selection cuts variation : $\pm 1 \sigma$ for all cuts applied;

Regeneration effects : variation of correction accoring to its statistical uncertainty;

KLOE reference frame orientation : uncertainty on the reference frame orientation;

Residual background subtraction : variation of subtracted background according to its uncertainty;

Fit range : range variation of $\pm 1 \tau_{S}$.

The total variations observed are summarized in table 1 .

Table 1: Summary of the systematic uncertainties. Units are $10^{-18} \mathrm{GeV}$. In all cases the systematics uncertainty is betweeen $30 \%$ and $50 \%$ of the corresponding statistical uncertainty.

\begin{tabular}{r|c|c|c|c|l} 
Parameter & Cut stability & Fit Range & Bkg subtraction & KLOE ref frame & Total \\
\hline$\Delta a_{0}$ & 1.1 & 2.4 & 1.3 & 1.0 & 3.1 \\
\hline$\Delta a_{X}$ & 0.3 & 0.3 & 0.4 & 0.2 & 0.6 \\
\hline$\Delta a_{Y}$ & 0.2 & 0.3 & 0.2 & 0.2 & 0.5 \\
\hline$\Delta a_{Z}$ & 0.2 & 0.2 & 0.4 & 0.4 & 0.6 \\
\hline
\end{tabular}

The final results on $\Delta a_{\mu}$ parameters are reported in the table 2. The expected sensitivity has been reached. The total error is fully dominated by the statistical uncertainty.

Table 2: Fit results. Errors include all the statistical fluctuations. The statistical errors are in the expected range. The fit $\chi^{2} / N d o F$ is $211.7 / 184$ corresponds to a p-value of $8 \%$. The second half of the table contains the correlation matrix coming from the fitting program.

\begin{tabular}{c|c||ccccc|} 
Par. & \multicolumn{1}{|c||}{ Fit output $\left(10^{-18} \mathrm{GeV}\right)$} & \multicolumn{5}{c}{ Correlation matrix } \\
$\Delta a_{0}$ & $\left(-6.0 \pm 7.7_{\text {stat }} \pm 3.1_{\text {syst }}\right)$ & 1.000 & 0.304 & -0.187 & 0.483 & -0.057 \\
\hline$\Delta a_{X}$ & $\left(0.9 \pm 1.5_{\text {stat }} \pm 0.6_{\text {syst }}\right)$ & 0.304 & 1.000 & -0.045 & 0.069 & -0.022 \\
\hline$\Delta a_{Y}$ & $\left(-2.0 \pm 1.5_{\text {stat }} \pm 0.5_{\text {syst }}\right)$ & -0.187 & -0.045 & 1.000 & -0.104 & 0.023 \\
\hline$\Delta a_{Z}$ & $\left(3.1 \pm 1.7_{\text {stat }} \pm 0.6_{\text {syst }}\right)$ & 0.483 & 0.069 & -0.104 & 1.000 & -0.045 \\
\hline
\end{tabular}

\section{Conclusions and future plans}

The continuation of the KLOE physics program with KLOE-2 [3] at an improved DAФNE machine is starting with a new beam interaction scheme [8] and with the inclusion of two pairs of electron-positron taggers for the study of the gamma-gamma physics: Low Energy Tagger [9] 
inside KLOE apparatus and High Energy Tagger [10] along the beam lines outside the KLOE detector. Several other upgrades for the detector are going to be installed: a pair of crystal calorimeters (CCALT) [11] near the interaction region to improve the angular acceptance for low- $\theta$ particles; a pair of tile calorimeters (QCALT) [12], covering the quadrupoles inside the KLOE detector and along the beam pipe, made of tungsten slabs and singly read-out scintillator tiles to improve the angular coverage for particles coming from the active volume of the DC; a small and light inner tracker (IT) [13] made of four planes of cylindrical GEM to improve the resolution of the vertex reconstruction around the interaction point and to increase the low- $\theta$ acceptance for charged particles.

The results presented in table 1 are expected to be improved with the KLOE- 2 data taking campaign. The sensitivity of $C P T$ and Lorentz Invariance tests will improve either by the increased statistics and by the new interaction region (IR) with IT. The IT will improve the resolution on the vertex position and the acceptance for low- $\theta$ tracks, the IR will imply a larger beam crossing angle (from 25 to $60 \mathrm{mrad}$ ) that will enhance the effect of asymmetry between the two kaons. The expected sensitivity should increase up to $10^{-19} \mathrm{GeV}$ for all the $\Delta a_{\mu}$ parameters.

\section{References}

[1] M. Adinolfi et al., KLOE Collaboration, Nucl. Inst. Meth. A 488 (2002) 51.

[2] M. Adinolfi et al., KLOE Collaboration Nucl. Inst. Meth. A 482 (2002) 363.

[3] G. Amelino-Camelia et al., Eur. Phys. J. C 68 (2010) 619 [arXiv:1003.3868 [hep-ex]].

[4] A. Einstein, B. Podolsky and N. Rosen, Phys. Rev. 47, 777 (1935).

[5] V. A. Kostelecky, Phys. Rev. D 64 (2001) 076001 [hep-ph/0104120].

[6] O. W. Greenberg, Phys. Rev. Lett. 89 (2002) 231602 [hep-ph/0201258].

[7] F. Ambrosino et al. [KLOE Collaboration], Phys. Lett. B 642 (2006) 315 [hep-ex/0607027].

[8] C. Milardi et al., DAFNE Collaboration, arXiv:1006.1487.

[9] D. Babusci et al., Nucl. Instrum. Meth. A 617, 81 (2010).

[10] F. Archilli, et al., Nucl. Instrum. Meth. A 617 (2010) 266.

[11] F. Happacher et al., Nucl. Phys. Proc. Suppl. 197, 215 (2009).

[12] M. Cordelli et al., Nucl. Instrum. Meth. A 617, 105 (2010).

[13] A. Balla, et al., Nucl. Instrum. Meth. A 628 (2011) 194 [arXiv:1003.3770 [physics.ins-det]]. 